\title{
OBSERVING SOFT X-RAY LINE EMISSION FROM THE INTERSTELLAR MEDIUM WITH X-RAY CALORIMETER ON A SOUNDING ROCKET
}

\author{
J. Zhang1 , B. Edwards', M. Juda1, R. Kelley², G. Madejski², D. McCammon', \\ H. Moseley ${ }^{2}$, M. Skinner ${ }^{1}$, R. Schoelkopf ${ }^{2}$, and A. Szymkowiak ${ }^{2}$ \\ 1 Physics Department, University - Madison, Madison, WI, USA \\ 2 NASA/Doddard Space Flight Center, Greenbelt, MD, USA
}

\begin{abstract}
We have been developing $X$-ray calorimeters that have high spectral resolution and high quantum efficiency. For an $\mathrm{X}$-ray calorimeter working at $0.1 \mathrm{~K}$, the energy resolution ideally can be as good as one $\mathrm{eV}$ for a practical detector. A detector with a resolution of $17 \mathrm{eV}$ FWHM at $6 \mathrm{keV}$ has been constructed. We expect to be able to improve this by a factor of two or more. With X-ray calorimeters flown on a sounding rocket, we should be able to observe soft $X$-ray line emission from the interstellar medium over the energy range 0.07 to $1 \mathrm{keV}$. Here we present a preliminary design for an X-ray calorimeter rocket experiment and the spectrum which might be observed from an equilibrium plasma. For later X-ray calorimeter sounding rocket experiments, we plan to add an aluminum foil mirror with collecting area of about 400 $\mathrm{cm}^{2}$ to observe line features from bright supernova remnants.
\end{abstract}

\section{INTRODUCTION}

The Soft X-ray Background (SXRB, E $<1.0 \mathrm{keV}$ ) has been studied for about twerity years, primarily with proportional counters. All the SXRB observations suggest that the major source of the SXRB is thermal emission from a hot, thin interstellar plasma of temperature $\sim 10^{6} \mathrm{~K}$ (Williamson et al.1974, McCammon et al. 1983, Marshall and Clark 1984). Observations of O VI and OVII (Jenkins 1974, Inoue 1979, Schnopper 1982, Rocchia 1984) also support a thermal model. This hot plasma should be rich in line emssion. Getting high resolution X-ray spectra of such a diffuse source is very difficult, but could add much information about its physical state and possibly its past history. $\mathrm{X}$-ray calorimeters have adequate resolution for this task, and a modest instrument can be constructed with enough throughput to make useful observations on a sounding rocket flight.

\section{INSTRUMENT}

The way in which the X-ray calorimeter works is that a photon is absorbed and its energy is converted to heat; the resulting temperature rise can be measured by detecting the resistance change of an ion-implanted silicon thermistor (Fig. 1.). More detailed descriptions are in McCammon et al. (1987) and Holt (1988). Since the energy resolution is limited by the heat capacity of an individual calorimeter, the collecting area can be increased by using multiple detectors. This also permits imaging operation if the detector array is used with a telescope.

We have been developing X-ray calorimeters at GSFC and at the University of Wisconsin. A detector with $17 \mathrm{eV}$ FWHM resolution at $6 \mathrm{keV}$ has been constructed. We expect 
to improve the energy resolution to $5 \mathrm{eV}$ for low energy $\mathrm{X}$-rays in the near future. Figures 1 and 2 show different views of a single detector element. Figure 3 is the electrical circuit used to run the calorimeters. Figure 4 is a sketch of the basic strucuture of a sounding rocket cryostat for $\mathrm{X}$-ray calorimeters. The required $0.1 \mathrm{~K}$ operating temperature is provided by an adiabatic demagnetization refrigerator (ADR). The salt pill (ferric ammonium alum) is fixed in a G-10 tube which is suspended by Kevlar fibers. A set of blocking filters is necessary to limit the shot noise in the detectors caused by low-energy photons from thermal infrared radiation and ultraviolet air glow. We plan to use four filters in series. Each one has $200 \AA$ aluminum evaporated on to a $1000 \AA$ parylene- $N$ substrate. The calculated net IR transmission of four filters is $<10^{-10}$. The dashed line in Figure 5 is the net $X$-ray transmission based on measurements of the individual filters. The $\mathrm{X}$-ray calorimeter has an intrinsic quantum efficiency of almost $100 \%$. The $X$-ray throughput is limited by the filter transmission and the detector array area. We are planning to observe emission from the interstellar medium (ISM) over about 1 steradian of the sky with $0.2 \mathrm{~cm}^{2}$ total detector area.

\section{OBSERVATION OF INTERSTELLAR MEDIUM EMISSION}

The solid curve in figure 5(a) is a calculated emission spectrum convolved with the expected $5 \mathrm{eV}$ resolution of the calorimeters. We have used the equilibruim plasma models of Raymond and Smith (1987), with solar abundances, and with the temperature and emission measure of each component chosen so that total B-band, C-band, and M-band count rates agree with the Wisconsin sky survey (McCammon 1983). Figure 5(b) shows a Monte Carlo simulation of a 300 second observation with the sounding rocket instrument described above. Even with such short observing time, the statistics are sufficient to permit a considerable amount of useful scientific analysis. For later X-ray calorimeter sounding rocket experiments, we plan to add an aluminum foil mirror with $400 \mathrm{~cm}^{2}$ collecting area (Serlemitsos 1981) to observe bright supernova remnants.

\section{REFERENCES}

Holt, S. S. 1988, invited talk in this colloquium.

Inoue, H. Koyama, K., Matsuoka, M., Ohashi, T., Tanaka, Y., and Tsuremi, H. 1979, Ap. J., (Letters), 227, L85.

Jenkins, E. B. and Meloy, D. A. 1974, Ap. J., (Letters), 293, L115.

Marshall, F. J. and Clark, G. W. 1984, Ap. J., 287, 633.

McCammon, D. Burrows, D. N., Sanders, W. T., and Kraushaar, W. L. 1983, Ap. J., 269, 107.

McCammon, D., Juda, M., Zhang, J., Holt, S. S., Kelley, R. C., Moseley, S. H., and

Szymkowiak, A. E. 1987, Proc. 18th Int. Conf. on Low Temperature Physics, Kyoto, 1987.

Japanese Journal of Applied Physics, Supplement, 26,3

Raymond, J. C. and Smith, B. W. 1977, Ap. J. Suppl., 35, 419 1987, private communication (update to Raymond and Smith 1977)

Rocchia, R.,Araud, M., Blondel, C., Cheron, C., Christy, J. C., Rothenflug, R., Schnopper, H. W., and Delvaille, J. P. 1984, Astron. Astrophys., 130, 53

Schnopper, H. W., Delvaille, J. P., Rocchia, R., Blondel, C.,.Cheron, C., Christy, J. C., Ducros, R., Koch, L., And Rothenflug, R 1982, Ap. J., 253, 131

Serlemitsos, P. J. et al., 1981, X-ray Astronomy in the 1980's, ed. S.Holt, NASA Technical Memorandum 83848, 441

Williamson, F. O., Sanders, W. T., Kraushaar, W. L., McCammon, D., Borken, R., and Burner, A. N. 1974, Ap. J., (Letters), 193, L133. 


\section{SIDE VIEW OF A SINGLE DETECTOR ELEMENT}

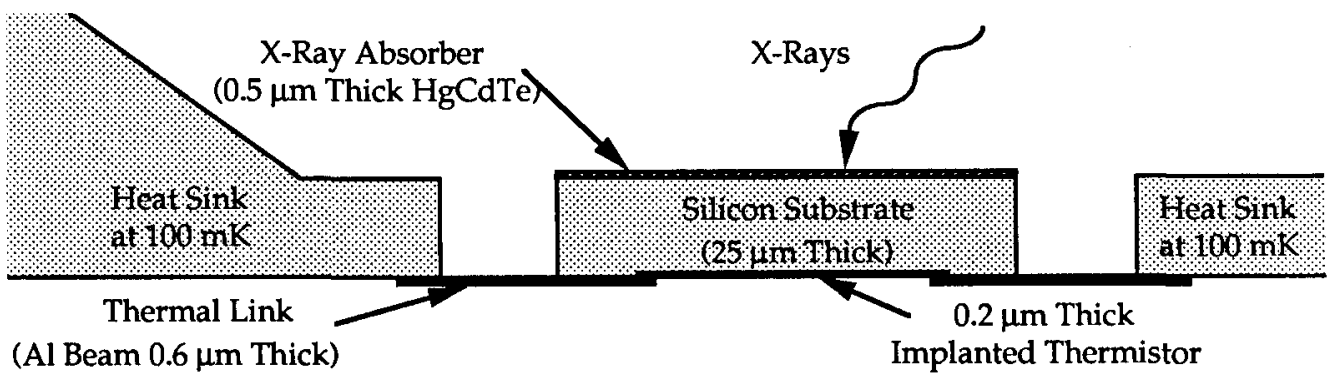

Figure 1. A side view of an X-ray calorimeter (not to scale). When an X-ray hits the absorber ( $\mathrm{HgCdTe}$ ), the photon energy is converted to thermal energy . The resulting temperature rise can be measured by the ion-implanted thermistor. Aluminum beams provide thermal and electrical links.

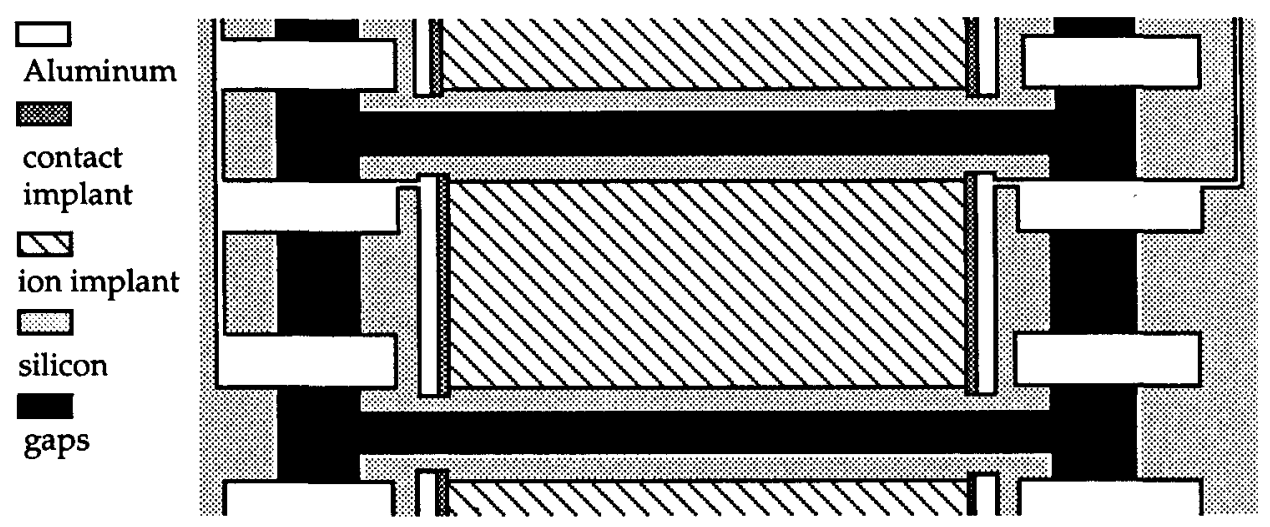

Figure 2. A single detector elemnt of a testing arraye

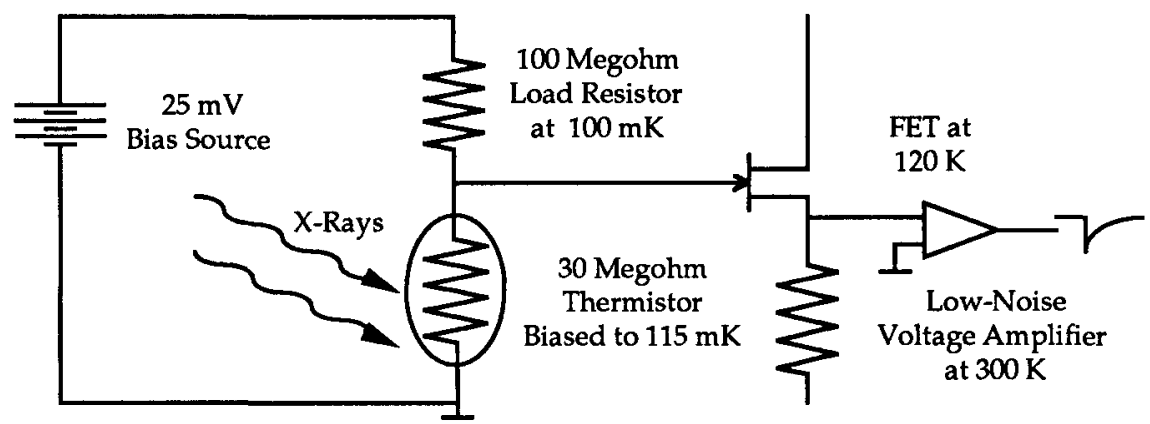

Figure 3. An electrical circuit used to run a calorimeter. 


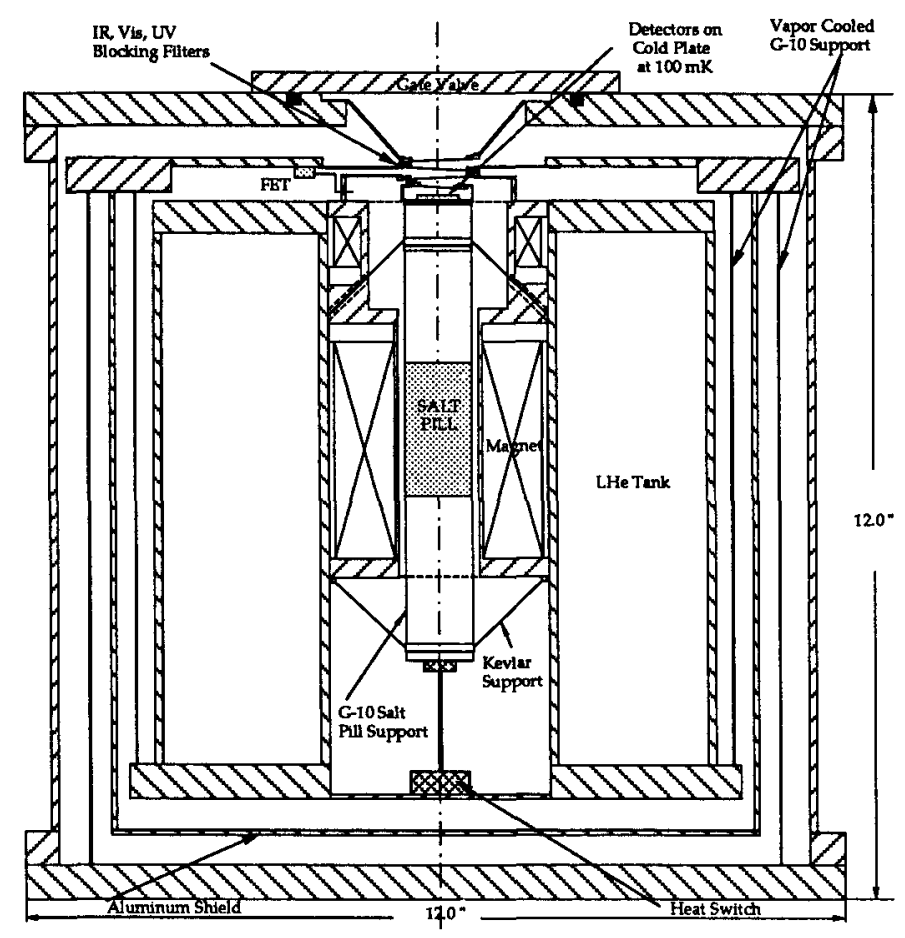

\section{X-Ray Calorimeter Sounding Rocket Cryostat}

Figure 4. The required $100 \mathrm{mK}$ operating temperature of a X-ray calorimeter is provided by an adiabatic demagnetization refrigerator. This shows the basic structure of the cryostat. The salt pill (ferric ammonium alum) is fixed in a G-10 tube which is suspended by kevlar fibers. A set of blocking filters is used to limit the shot noise in the detectors caused by low-energy photons from thermal infrared radiation and ultraviolet air glow. The G-10 shell supports for the liquid helium dewar are vapor cooled.

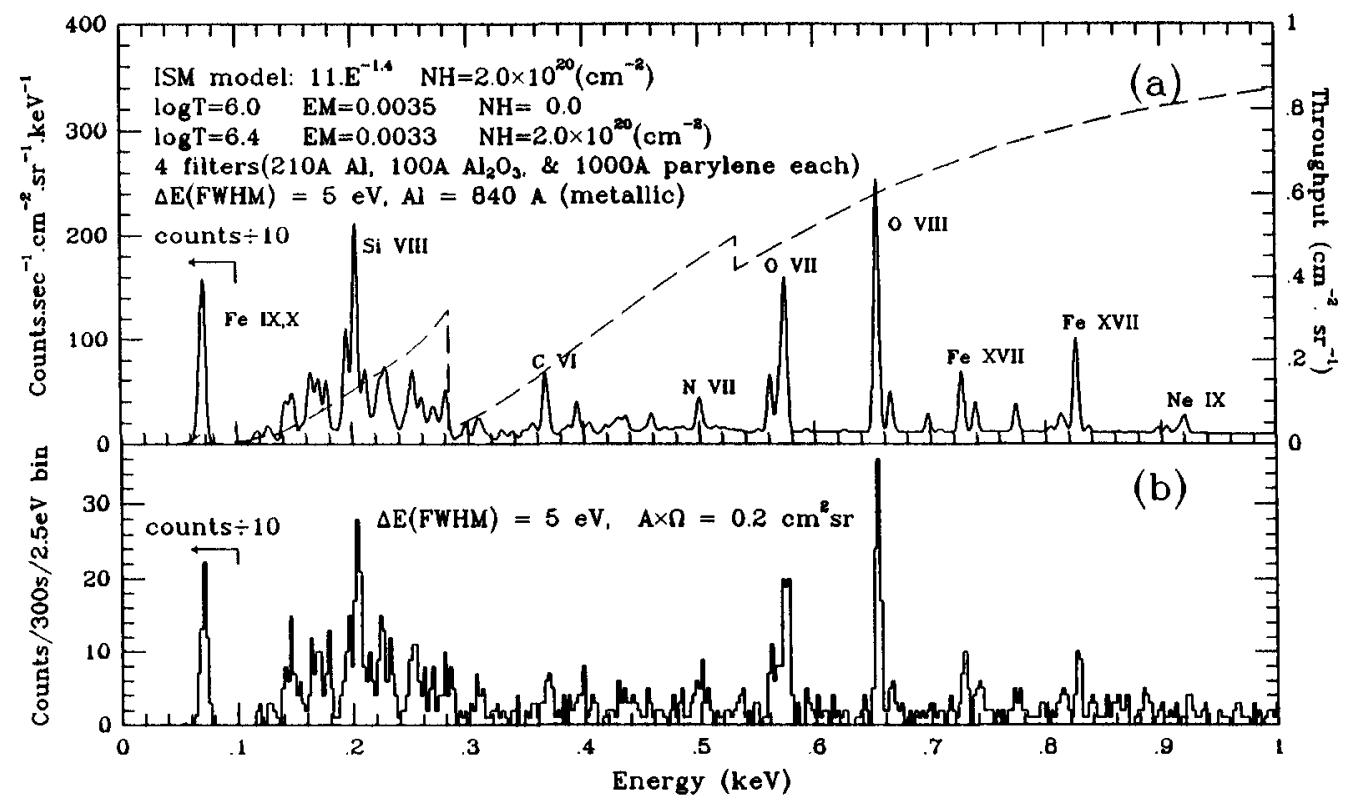

Figure 5. (a) A calculated emissison spectrum convolved with the expected $5 \mathrm{eV}$ resolution of the calorimeters. We have used equilibrium plasma models of Raymond and Smith (1987), with solar abundance, and with the temperature and emission measure of each component chosen so that total B-band, C-band, and M-band count rates agree with the Wisconsin sky survey (McCammon 1983). The dashed line is the instrument response (net X-ray transmission of four filters). (b) A monte Carlo simulation of 300 second observation with sounding rocket instrument described in the text. 